\title{
Hybrid synchronization of n-scroll Chua and Lur'e chaotic systems via backstepping control with novel feedback
}

\author{
SURESH RASAPPAN and SUNDARAPANDIAN VAIDYANATHAN
}

\begin{abstract}
This paper investigates the backstepping control design with novel feedback input approach for controlling chaotic systems to guarantee the complete synchronization as well as the anti-synchronization of chaotic systems, viz. n-scroll Chua (K. Wallace et.al. 2001) and Lur'e chaotic systems. Our theorems on hybrid synchronization for n-scroll Chua and Lur'e (J.Suyken et.al. 1997) chaotic systems is established using Lyapunov stability theory. Based on the Lyapunov function, the backstepping control is determined to tune the controller gain based on the precalculated feedback control inputs. The backstepping scheme is recursive procedure that links the choice of a Lyapunov function with the design of a controller and guarantees global stability performance of strict-feedback chaotic systems. Since the Lyapunov exponents are not required for these calculations, the backstepping control method is effective and convenient to synchronize the chaotic systems. Mainly this technique gives the flexibility to construct a control law. Numerical simulations are also given to illustrate and validate the hybrid synchronization results derived in this paper.
\end{abstract}

Key words: chaos, hybrid-synchronization, n-scroll Chua system, Lur'e system, backstepping control

\section{Introduction}

Chaos is a strange random aggregate of responses to internal and external stimuli in dynamic systems. It is highly sensitive towards initial conditions. That is to say, chaotic systems starting off from very similar initial states can develop into radically divergent ways. Such sensitive dependence is often referred to as the Butterfly effect. The theories and methods developed for controlling nonlinear systems could be utilized for synchronization of chaotic systems. In general, synchronization research has been focused on two areas. The first one work with the state observers, where the main applications pertain to the synchronization of nonlinear oscillators. The second one is the use of control

The Authors are with Vel Tech Dr. RR \& Dr. SR Technical University, \# 42 Avadi - Vel Tech Road, Avadi, Chennai- 600062, Tamilnadu, India. R. Suresh, the corresponding author, is with Department of Mathematics, e-mail: mrpsuresh83@gmail.com. V. Sundarapandian is with Research and Development Centre.

Received 10.05.2012. Revised 20.06.2012. 
laws, which allows to achieve the synchronization between nonlinear oscillators, with different structure and order.

The synchronization of chaotic system was first researched by Yamada and Fujisaka [1] with subsequent work by Pecora and Carroll [2, 3]. The synchronization of chaos is one way of explaining sensitive dependence on initial conditions $[4,5]$. It has been established that the synchronization of two chaotic systems, that identify the tendency of two or more systems are coupled together to undergo closely related motions. The problem of chaos synchronization is to design a coupling between the two systems such that the chaotic time evaluation becomes ideal. The output of the slave system asymptotically follows the output of the master system i.e. the output of the master system controls the slave system.

The synchronization for chaotic systems has been widespread to the scope, such as generalized synchronization [6], phase synchronization [7], lag synchronization, projective synchronization [8], generalized projective synchronization [9, 10, 11, 12] and even anti-synchronization. The property of anti-synchronization establish a predominating phenomenon in symmetrical oscillators, in which the state vectors have the same absolute values but opposite signs. When synchronization and anti-synchronization coexist, simultaneously, in chaotic systems, the synchronization is called hybrid synchronization $[13,14,15]$.

Various control methods have been developed in order to synchronize chaotic systems which includes for ensuring the control and synchronization of such systems and have demonstrated their potential applications in various fields including chaos generator design, such as secure communication [16, 17], physical systems [18], chemical reaction [19], ecological systems [20], information science [21], energy resource systems [22], ghostburster neurons [23], bi-axial magnet models [24], neuronal models [25, 26], IR epidemic models with impulsive vaccination [27] and predicting the influence of solar wind to celestial bodies [28], etc. So far a variety of impressive approaches have been proposed for the synchronization of the chaotic systems such as the OGY method [29], sampled feedback synchronization method, time delay feedback method [30], adaptive design method [31, 32, 33], sliding mode control method [34, 35], active control method [36, 37], etc.

Recently, backstepping method has been developed for designing controllers to control the chaotic systems $[38,39]$. A common concept of the method is the synchronization of chaotic system. The backstepping method is based on the mathematical model of the examined system, introducing new variables into it in a form depending on the state variables, controlling parameters, and stabilizing functions. The difficult work of synchronizing the chaotic system is to remove nonlinearities done in the system and influencing the stability of state operation. The use of backstepping method creates an additional non-linearity and eliminates undesirable nonlinearities from the system.

In this paper, backstepping control design with novel feedback input approach is proposed. This approach is a systematic design approach and guarantees global stability of the n-scroll Chua (K. Wallace et.al. [40]) and Lur'e (J.Suyken et.al. [41, 42] ) chaotic systems. Based on the Lyapunov function, the backstepping control is determined to 
tune the controller gain based on the precalculated feedback control inputs. We organize this paper as follows. In Section 2, we present the methodology of chaos hybrid synchronization by backstepping control method. In Section 3, we give a description of the chaotic systems discussed in this paper. In Section 4, we demonstrate the chaos hybrid synchronization of identical $\mathrm{n}-$ scroll Chua systems [40]. In Section 5, we demonstrate the chaos hybrid synchronization of identical Lur'e systems In Section 6, we demonstrate the chaos hybrid synchronization of $\mathrm{n}-$ scroll Chua and Lur'e systems. In Section 7 , we summarize the results obtained in this paper.

\section{The problem statement and methodology}

In general, the two dynamic systems in hybrid synchronization are called the master and slave system, respectively. Well designed controller makes the trajectory of the slave system track the trajectory of the master system, that is, the two systems are synchronous.

Consider the dynamics of nonlinear systems whose trajectories have chaotic attractor:

$$
\begin{aligned}
\dot{x}_{1} & =F_{1}\left(x_{1}, x_{2}, \ldots, x_{n}\right) \\
\dot{x}_{2} & =F_{2}\left(x_{1}, x_{2}, \ldots, x_{n}\right) \\
\dot{x}_{3} & =F_{3}\left(x_{1}, x_{2}, \ldots, x_{n}\right) \\
\vdots & \\
\dot{x}_{n} & =F_{n}\left(x_{1}, x_{2}, \ldots, x_{n}\right)
\end{aligned}
$$

where $x(t) \in \mathbb{R}$ is state vectors of the system. Assume that the master system is described by (1) and the slave system which is coupled to (1) with the controller $u$ is defined by:

$$
\begin{aligned}
\dot{y}_{1} & =G_{1}\left(y_{1}, y_{2}, \ldots, y_{n}\right)+u_{1}(t) \\
\dot{y}_{2} & =G_{2}\left(y_{1}, y_{2}, \ldots, y_{n}\right)+u_{2}(t) \\
\dot{y}_{3} & =G_{3}\left(y_{1}, y_{2}, \ldots, y_{n}\right)+u_{3}(t) \\
\vdots & \\
\dot{y}_{n} & =G_{n}\left(y_{1}, y_{2}, \ldots, y_{n}\right)+u_{n}(t)
\end{aligned}
$$

where $x(t) \in \mathbb{R}^{n}$ is a state vectors of the system and $F_{i}, G_{i}(i=1,2,3, \ldots n)$ are linear and nonlinear functions with inputs from systems (1) and (2). If $F_{i}$ equals to $G_{i}$, then the systems states are identical, otherwise the systems' states are non identical. The chaotic systems (1) and (2) depends not only on state variables but also on time $t$. The problem is to analyze the synchronization between two chaotic systems and to transform it to another problem, namely how to choose the control law $u_{i}, i=1,2,3, \ldots, n$ to make the difference between these two systems, $e_{i}, i=1,2,3, \ldots, n$ converge to zero with the time increasing. 
In order to observe the synchronization behavior in master and slave systems, we have introduced the control functions $u_{i}, i=1,2,3, \ldots, n$ for the purpose of synchronizing the master and slave systems in spite of a different chaotic systems which is the extreme case of master/slave mismatch. To estimate the control functions, we subtract (1) from (2), and define the hybrid synchronization error system as the differences between the slave system (2) and the controlled master system. Let us define the error variables between the slave system (2) that is to be controlled and the controlling master system (1) as

$$
e_{i}=y_{i} \pm x_{i}, i=1,2,3, \ldots, n .
$$

Then the synchronization error dynamics is obtained as

$$
\begin{aligned}
\dot{e}_{1} & =G_{1}\left(y_{1}, y_{2}, \ldots, y_{n}\right)-F_{1}\left(x_{1}, x_{2}, \ldots, x_{n}\right)+u_{1}(t) \\
\dot{e}_{2} & =G_{2}\left(y_{1}, y_{2}, \ldots, y_{n}\right)+F_{2}\left(x_{1}, x_{2}, \ldots, x_{n}\right)+u_{2}(t) \\
\dot{e}_{3} & =G_{3}\left(y_{1}, y_{2}, \ldots, y_{n}\right)-F_{3}\left(x_{1}, x_{2}, \ldots, x_{n}\right)+u_{3}(t) \\
\vdots & \\
\dot{e}_{n} & =G_{n}\left(y_{1}, y_{2}, \ldots, y_{n}\right)-F_{n}\left(x_{1}, x_{2}, \ldots, x_{n}\right)+u_{n}(t) .
\end{aligned}
$$

The hybrid synchronization error system controls a controlled chaotic system with control input $u_{i}, i=1,2,3, \ldots, n$ as a function of the error states $e_{1}, e_{2}, e_{3}, \ldots . ., e_{n}$. That means the systematic feedbacks so as to stabilize the error dynamics (3), $e_{1}, e_{2}, e_{3}, \ldots . ., e_{n}$ converge to zero as time $t \rightarrow \infty$. This implies that the controllers $u_{i}, i=1,2,3, \ldots, n$ should be designed so that the two chaotic systems can be synchronized. Mathematically this means

$$
\lim _{t \longrightarrow \infty}\|e(t)\|=0 .
$$

Backstepping design is recursive method which can guarantee global stable performance of strict-feedback nonlinear systems. By using the backstepping design, at the $i^{\text {th }}$ step, the $i^{\text {th }}$ order subsystem is stabilized with respect to a Lyapunov function $V_{i}$, by the design of virtual control $\alpha_{i}$ and a control input function $u_{i}$.

We consider the stability problem of the system

$$
\dot{e}_{1}=G_{1}\left(y_{1}, y_{2}, \ldots, y_{n}\right)-F_{1}\left(x_{1}, x_{2}, \ldots, x_{n}\right)+u_{1}(t)
$$

where $u_{1}$ is control input, which is the function of the error state vectors $e_{i}$, and the state variables $x(t) \in \mathbb{R}^{n}, y(t) \in \mathbb{R}^{n}$. As long as this feedback stabilize the system (4) converge to zero as the time $t \rightarrow \infty$, where $e_{2}=\alpha_{1}\left(e_{1}\right)$ is regarded as virtual controller.

For the design of $\alpha_{1}\left(e_{1}\right)$ to stabilize the subsystem (4), we consider the Lyapunov function defined by

$$
V_{1}\left(e_{1}\right)=e_{1}^{T} P_{1} e_{1}
$$

The derivative of $V_{1}$ is

$$
\dot{V}_{1}=-e_{1}^{T} Q_{1} e_{1}
$$


where $Q_{1}$ is a positive definite matrix. Then $\dot{V}_{1}$ is a negative definite function on $\mathbb{R}^{n}$. Thus by Lyapunov stability theory [43] the error dynamics (4) is asymptotically stable. The virtual control $e_{2}=\alpha_{1}\left(e_{1}\right)$ and the state feedback input $u_{1}$ makes the system (4) asymptotically stable. The function $\alpha_{1}\left(e_{1}\right)$ should be estimated while $e_{2}$ is considered as controller.

The error between $e_{2}$ and $\alpha_{1}\left(e_{1}\right)$ is

$$
w_{2}=e_{2}-\alpha_{1}\left(e_{1}\right) .
$$

Consider $\left(e_{1}, w_{2}\right)$ subsystem given by

$$
\begin{aligned}
\dot{e}_{1} & =G_{1}\left(y_{1}, y_{2}, \ldots, y_{n}\right)-F_{1}\left(x_{1}, x_{2}, \ldots, x_{n}\right) \\
\dot{w_{2}} & =G_{2}\left(y_{1}, y_{2}, \ldots, y_{n}\right)+F_{2}\left(x_{1}, x_{2}, \ldots, x_{n}\right)-\dot{\alpha}_{1}\left(e_{1}\right)+u_{2} .
\end{aligned}
$$

Consider $e_{3}$ as a virtual controller in system (8), assume that it is equal to $\alpha_{2}\left(e_{1}, w_{2}\right)$ and it makes the system (8) asymptotically stable. Consider the Lyapunov function defined by

$$
V_{2}\left(e_{1}, w_{2}\right)=V_{1}\left(e_{1}\right)+w_{2}^{T} P_{2} w_{2} .
$$

The derivative of $V_{2}$ is

$$
\dot{V}_{2}=-e_{1}^{T} Q_{1} e_{1}-w_{2}^{T} Q_{2} w_{2}<0
$$

where $Q_{1}, Q_{2}$ are positive definite matrices. Then $\dot{V}_{2}$ is a negative definite function on $\mathbb{R}^{n}$. Thus by Lyapunov stability theory the error dynamics (8) is asymptotically stable. The virtual control $e_{3}=\alpha_{2}\left(e_{1}, w_{2}\right)$ and the state feedback input $u_{2}$ make the system (8) asymptotically stable. For the $n_{t h}$ state of the error dynamics, define the error variable $w_{n}$ as

$$
w_{n}=e_{n}-\alpha_{n-1}\left(e_{1}, w_{2}, w_{2}, \ldots, w_{n}\right) .
$$

Consider $\left(e_{1}, w_{2}, w_{2}, \ldots, w_{n}\right)$ subsystem given by

$$
\begin{aligned}
\dot{e}_{1}= & G_{1}\left(y_{1}, y_{2}, \ldots, y_{n}\right)-F_{1}\left(x_{1}, x_{2}, \ldots, x_{n}\right) \\
\dot{w_{2}}= & G_{2}\left(y_{1}, y_{2}, \ldots, y_{n}\right)+F_{2}\left(x_{1}, x_{2}, \ldots, x_{n}\right)-\dot{\alpha}_{1}\left(e_{1}\right) \\
\vdots & \\
\dot{w_{n}=} & G_{n}\left(y_{1}, y_{2}, \ldots, y_{n}\right)-F_{n}\left(x_{1}, x_{2}, \ldots, x_{n}\right) \\
& -\dot{\alpha}_{n-1}\left(e_{1}, w_{2}, w_{3}, \ldots, w_{n}\right)+u_{n} .
\end{aligned}
$$

Consider the Lyapunov function defined by

$$
V_{n}\left(e_{1}, w_{2}, w_{3}, \ldots, w_{n}\right)=V_{n-1}\left(e_{1}, w_{2}, w_{3}, \ldots, w_{n-1}\right)+w_{n}^{T} P_{n} w_{n} .
$$

The derivative of $V_{n}$ is

$$
\dot{V}_{n}=-e_{1}^{T} Q_{1} e_{1}-w_{2}^{T} Q_{2} w_{2}-\ldots .-w_{n}^{T} Q_{n} w_{n}<0
$$


where $Q_{1}, Q_{2}, Q_{3}, \ldots, Q_{n}$ are positive definite matrices. Then $\dot{V}_{n}$ is a negative definite function on $\mathbb{R}^{n}$. Thus by Lyapunov stability theory the error dynamics (12) is stable. The virtual control $e_{n}=\alpha_{n-1}\left(e_{1}, w_{2}, w_{3}, \ldots, w_{n-1}\right)$ and the state feedback input $u_{n}$ makes the system (12) asymptotically stable. Thus by Lyapunov stability theory, the error dynamics (3) is globally exponentially stable for all initial conditions $e(0) \in \mathbb{R}^{n}$. Hence, the states of the master and slave systems are globally and exponentially synchronized.

\section{The system description}

The first system in which we are interested is the n-scroll Chua system which is an improved model of chaotic system introduced by K. Wallace et.al. [40]. The second system is derived from Lur'e system. Both systems can be derived from simplified, generic electrical circuit.

\subsection{The n-scroll Chua system}

The dynamical equation of $\mathrm{n}$-scroll Chua system with sine function [40], is given by

$$
\begin{aligned}
& \dot{x}_{1}=\alpha\left(x_{2}-f\left(x_{1}\right)\right) \\
& \dot{x}_{2}=x_{1}-x_{2}+x_{3} \\
& \dot{x}_{3}=-\beta x_{2}
\end{aligned}
$$

where $x_{1}, x_{2}, x_{3}$ are state variables and $f\left(x_{1}\right)$, given by

$$
f\left(x_{1}\right)=\left\{\begin{array}{clc}
\frac{b \pi}{2 a}\left(x_{1}-2 a c\right) & \text { if } & x_{1} \geqslant 2 a c \\
-b \sin \left(\frac{\pi x_{1}}{2 a}+d\right) & \text { if } & -2 a c \leqslant x_{1} \leqslant 2 a c \\
\frac{b \pi}{2 a}\left(x_{1}+2 a c\right) & \text { if } & x_{1} \leqslant-2 a c
\end{array}\right.
$$

is the piecewise linear function and is the only nonlinearity in the system. A sine function is couched to obtain the nonlinearity needed for generating chaos in Chua system. $\alpha, \beta$, $a, b, c$ and $d$ are positive constants. When

$$
\alpha=10.814, \beta=14.0, a=1.3, b=0.11
$$

2-scroll, 3-scroll, 4-scroll and 6-scroll attractors are generated with $c=1,2,3$, and 5 respectively, as depicted in Fig. 1(a)-(d). A maximum of six scroll can be observed.

\subsection{Lur'e chaotic system}

The dynamical equation of Lur'e system (J. Suyken et.al. [41, 42]) with sign function is given by 

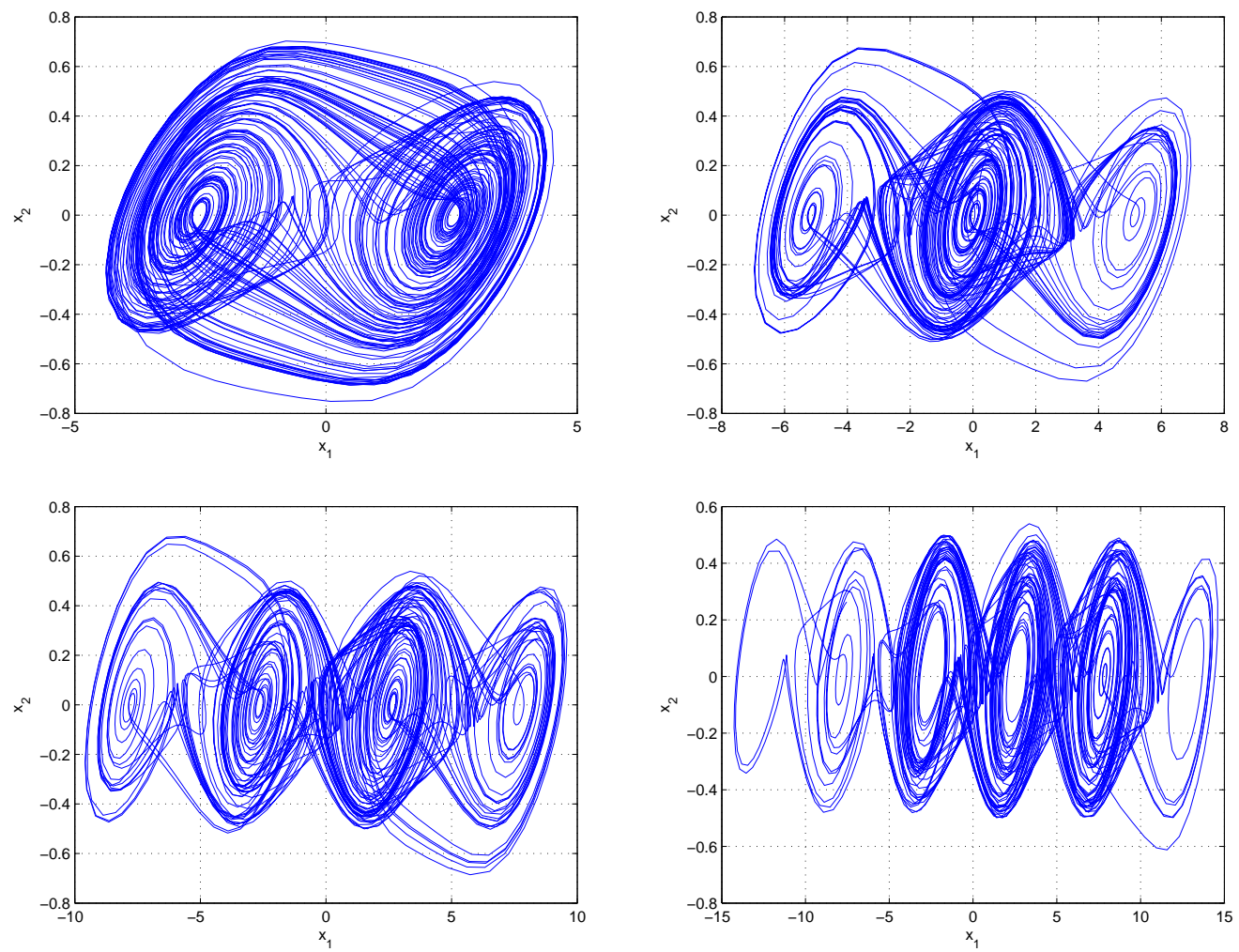

Figure 1. (a). Phase orbit of 2-scroll Chua system when $c=1$, (b). Phase orbit of 3-scroll Chua system when $c=2$, (c). Phase orbit of 4-scroll Chua system when $c=3$, (d). Phase orbit of 6-scroll Chua system when $c=5$.

$$
\begin{aligned}
& \dot{x}_{1}=x_{2} \\
& \dot{x}_{2}=x_{3} \\
& \dot{x}_{3}=a x_{1}+b x_{2}+c x_{3}+12 \phi\left(x_{1}\right)
\end{aligned}
$$

where $x_{1}, x_{2}, x_{3}$ are state variables. Function $\phi\left(x_{1}\right)$ is given by

$$
\phi\left(x_{1}\right)=\left\{\begin{array}{ccc}
k x_{1} & \text { if } & \left|x_{1}\right| \geqslant \frac{1}{k} \\
\operatorname{sign}\left(x_{1}\right) & & \text { otherwise }
\end{array}\right.
$$

where $a, b, c$ and $k$ are positive real constants. When

$$
a=-7.4, b=-4.1, c=-1, k=3.6
$$

the chaotic attractors are generated as shown in Fig. 2. 

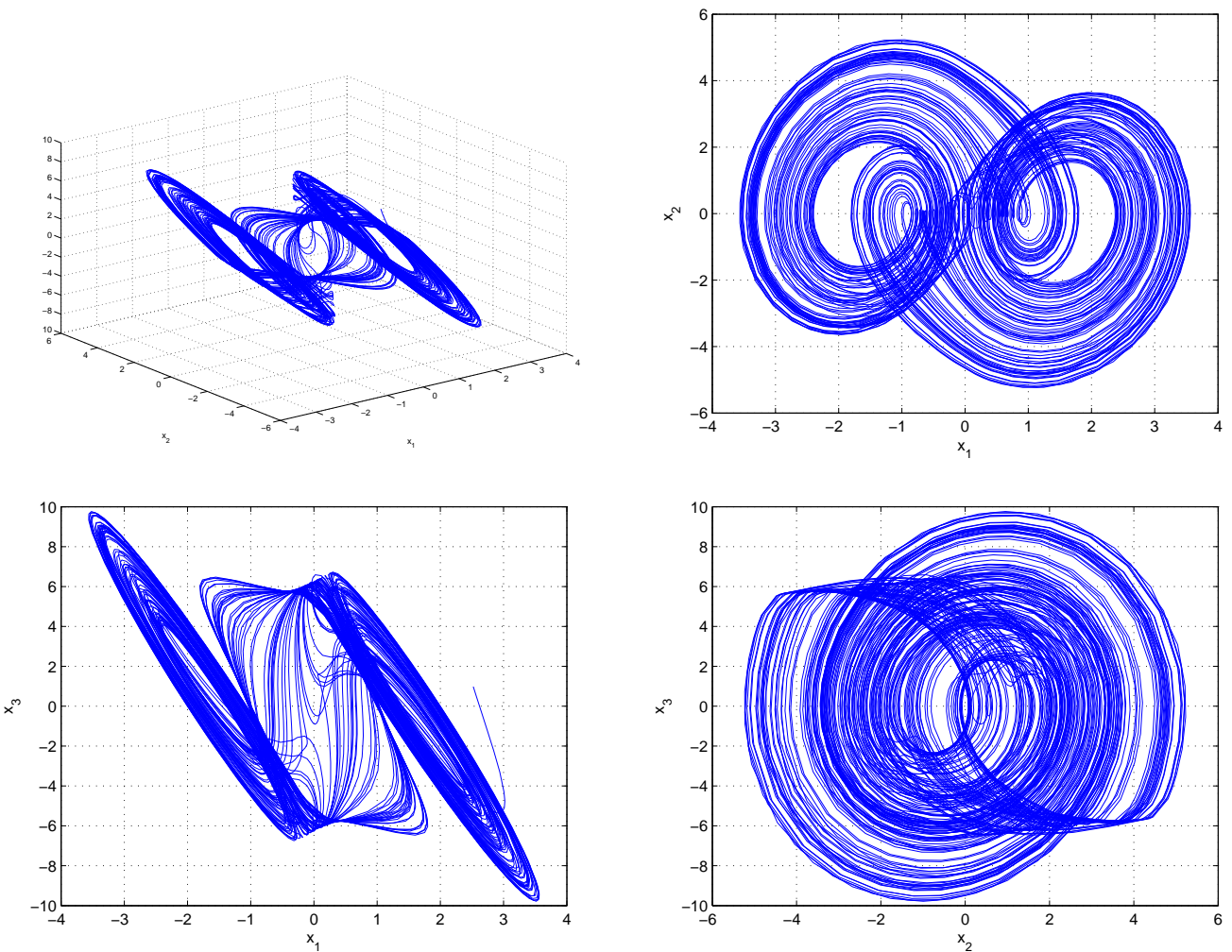

Figure 2. Phase orbits of Lur'e system.

\section{The hybrid synchronization of identical n-scroll Chua systems via backstepping control design with novel feedback}

In this section we apply the backstepping method with novel feedback function for the hybrid synchronization of identical Chua systems [40]. The master system is described by the chaotic n-scroll Chua's system dynamics (15) with parameters (16) which allows generating 2-scroll, 3-scroll, 4-scroll and 6-scroll attractors.The salve system is also described the chaotic n-scroll Chua's system dynamics

$$
\begin{aligned}
& \dot{y}_{1}=\alpha\left(y_{2}-f\left(y_{1}\right)\right)+u_{1} \\
& \dot{y}_{2}=y_{1}-y_{2}+y_{3}+u_{2} \\
& \dot{y}_{3}=-\beta y_{2}+u_{3}
\end{aligned}
$$


where $f\left(y_{1}\right)$ is given by

$$
f\left(y_{1}\right)=\left\{\begin{array}{clc}
\frac{b \pi}{2 a}\left(y_{1}-2 a c\right) & \text { if } & y_{1} \geqslant 2 a c \\
-b \sin \left(\frac{\pi y_{1}}{2 a}+d\right) & \text { if } & -2 a c \leqslant y_{1} \leqslant 2 a c \\
\frac{b \pi}{2 a}\left(y_{1}+2 a c\right) & \text { if } & y_{1} \leqslant-2 a c
\end{array}\right.
$$

and $y(t)(i=1,2,3) \in \mathbb{R}$ is a state vectors of the system. Let us define the error variables between the slave system (19) that is to be controlled and the controlling master system (15) as

$$
e_{i}=y_{i} \pm x_{i}, i=1,2,3, \ldots, n \text {. }
$$

The error dynamics is obtained as

$$
\begin{aligned}
& \dot{e}_{1}=\alpha y_{2}-\alpha x_{2}-\alpha\left[f\left(y_{1}\right)-f\left(x_{1}\right)\right]+u_{1} \\
& \dot{e}_{2}=y_{1}+x_{1}-e_{2}+y_{3}+x_{3}+u_{2} \\
& \dot{e}_{3}=-\beta y_{2}+\beta x_{2}+u_{3} .
\end{aligned}
$$

We introduce the backstepping procedure to design the controller $u_{i}, i=1,2,3$ where $u_{i}$ are the control feedbacks. As long as these feedbacks stabilize system (20), the error converges to zero as the time $t \rightarrow \infty$.

Firstly we consider the stability of the system

$$
\dot{e}_{1}=\alpha y_{2}-\alpha x_{2}-\alpha\left[f\left(y_{1}\right)-f\left(x_{1}\right)\right]+u_{1}
$$

where $e_{2}$ is regarded as virtual controller. The Lyapunov function is defined by

$$
V_{1}\left(e_{1}\right)=\frac{1}{2} e_{1}^{2}
$$

and its derivative is as follows

$$
\dot{V}_{1}=e_{1}\left(-e_{2}-e_{1}\right) \text {. }
$$

Assume the controller $e_{2}=\alpha_{1}\left(e_{1}\right)$. If $\alpha_{1}\left(e_{1}\right)=0$ and

$$
u_{1}=\alpha x_{2}-\alpha y_{2}+\alpha\left(f\left(y_{1}\right)-f\left(x_{1}\right)\right)-e_{2}-e_{1}
$$

then

$$
\dot{V}_{1}=-e_{1}^{2}
$$

which is negative definite function. The recursive feedback $u_{1}$ and $\alpha_{1}\left(e_{1}\right)$ makes the system (20) asymptotically stable. Function $\alpha_{1}\left(e_{1}\right)$ is the estimating function when $e_{2}$ is considered as a controller. The error between $e_{2}$ and $\alpha_{1}\left(e_{1}\right)$ is

$$
w_{2}=e_{2}-\alpha_{1}\left(e_{1}\right)
$$


Consider $\left(e_{1}, w_{2}\right)$ subsystem given by

$$
\begin{aligned}
\dot{e}_{1} & =-e_{2}-e_{1} \\
\dot{w_{2}} & =y_{1}+x_{1}-e_{2}+y_{3}+x_{3}+u_{2}
\end{aligned}
$$

and $e_{3}$ as a virtual controller in system (25). Assume that if it is equal to $\alpha_{2}\left(e_{1}, w_{2}\right)$, then it makes system (25) asymptotically stable. Now the Lyapunov function is defined by

$$
V_{2}\left(e_{3}, w_{2}\right)=V_{1}\left(e_{1}\right)+\frac{1}{2} w_{2}^{2} .
$$

The derivative of $V_{2}\left(e_{3}, w_{2}\right)$ is

$$
\dot{V}_{2}=-e_{1}^{2}+w_{2}^{2}\left(-w_{2}+e_{3}\right) .
$$

If $\alpha_{2}\left(e_{1}, w_{2}\right)=0$ and

$$
u_{2}=e_{1}-y_{1}-x_{1}-y_{3}-x_{3}+e_{3}
$$

then

$$
\dot{V}_{2}=-e_{1}^{2}-w_{2}^{2}
$$

which is negative definite function. The recursive control $u_{2}$ and $\alpha_{2}\left(e_{1}, w_{2}\right)$ makes the system (25) asymptotically stable. Define the error variable $w_{3}$ as

$$
w_{3}=e_{3}-\alpha_{2}\left(e_{1}, w_{2}\right) .
$$

Consider $\left(e_{1}, w_{2}, w_{3}\right)$ subsystem given by

$$
\begin{aligned}
\dot{e}_{1} & =-e_{1}-w_{2} \\
\dot{w_{2}} & =e_{1}-w_{2}+w_{3} \\
\dot{w_{3}} & =\beta y_{2}+\beta x_{2}+u_{3}
\end{aligned}
$$

and the Lyapunov function defined by

$$
V_{3}\left(e_{1}, w_{2}, w_{3}\right)=V_{2}\left(e_{1}, w_{2}\right)+\frac{1}{2} w_{3}^{2} .
$$

The derivative of $V_{3}\left(e_{3}, w_{2}, w_{3}\right)$ is

$$
\dot{V}_{3}=-e_{1}^{2}-w_{2}^{2}+w_{3}\left(w_{2}-\beta y_{2}+\beta x_{2}+u_{3}\right) .
$$

If $u_{3}=\beta y_{2}-\beta x_{2}-w_{2}-w_{3}$ then

$$
\dot{V}_{3}=-e_{1}^{2}-w_{2}^{2}-w_{3}^{2}
$$

which is negative definite function. The recursive feedback $u_{3}$ makes the system (29) asymptotically stable. Thus, by Lyapunov stability theory [43], the error dynamics (20) is globally exponentially stable. Hence, we obtain the following result. 
Theorem 1 The identical n-scroll Chua's systems (15) and (19) are globally and exponentially hybrid synchronized with the backstepping controls

$$
\begin{aligned}
& u_{1}=\alpha x_{2}-\alpha y_{2}+\alpha\left(f\left(y_{1}\right)-f\left(x_{1}\right)\right)-e_{2}-e_{1} \\
& u_{2}=e_{1}-y_{1}-x_{1}-y_{3}-x_{3}+e_{3} \\
& u_{3}=\beta y_{2}-\beta x_{2}-w_{2}-w_{3} .
\end{aligned}
$$

\section{Numerical simulation}

For the numerical simulations, the fourth order Runge-Kutta method is used to solve the system of differential equations (15) and (19) with the backstepping controls $u_{1}, u_{2}$ and $u_{3}$ given by (32). The parameters of the systems (15) and (19) are

$$
\alpha=10.814, \beta=14.0, a=1.3, b=0.11, c=3 .
$$

Initial value of the master system (15) are chosen as

$$
x_{1}(0)=0.125, x_{2}(0)=0.625 x_{3}(0)=0.941
$$

and slave system (19) are chosen as

$$
y_{1}(0)=0.874, y_{2}(0)=0.465, y_{3}(0)=0.596
$$

Fig. 3 (a) and (b) depict the hybrid synchronization of identical n-scroll Chua's systems (15) and (19).

\section{The hybrid synchronization of identical Lur'e systems via backstepping control design with novel feedback}

In this section we apply the backstepping method with novel feedback function for the hybrid synchronization of identical Lur'e systems [41, 42]. The master system is described by the chaotic Lur'e dynamics (17) with parameters (18).

The slave system also described by Lur'e dynamic sa follows

$$
\begin{aligned}
& \dot{y}_{1}=y_{2}+u_{1} \\
& \dot{y}_{2}=y_{3}+u_{2} \\
& \dot{y}_{3}=e y_{1}+f y_{2}+g y_{3}+12 \phi\left(y_{1}\right)+u_{3}
\end{aligned}
$$

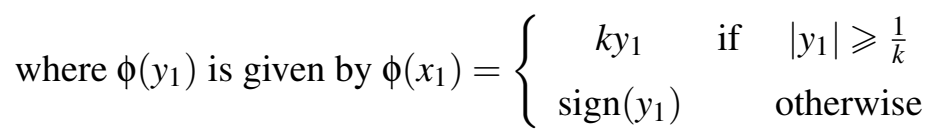
$y(t)(i=1,2,3) \in \mathbb{R}^{3}$ is a state vectors of the system. Let us define the error variables 
a)

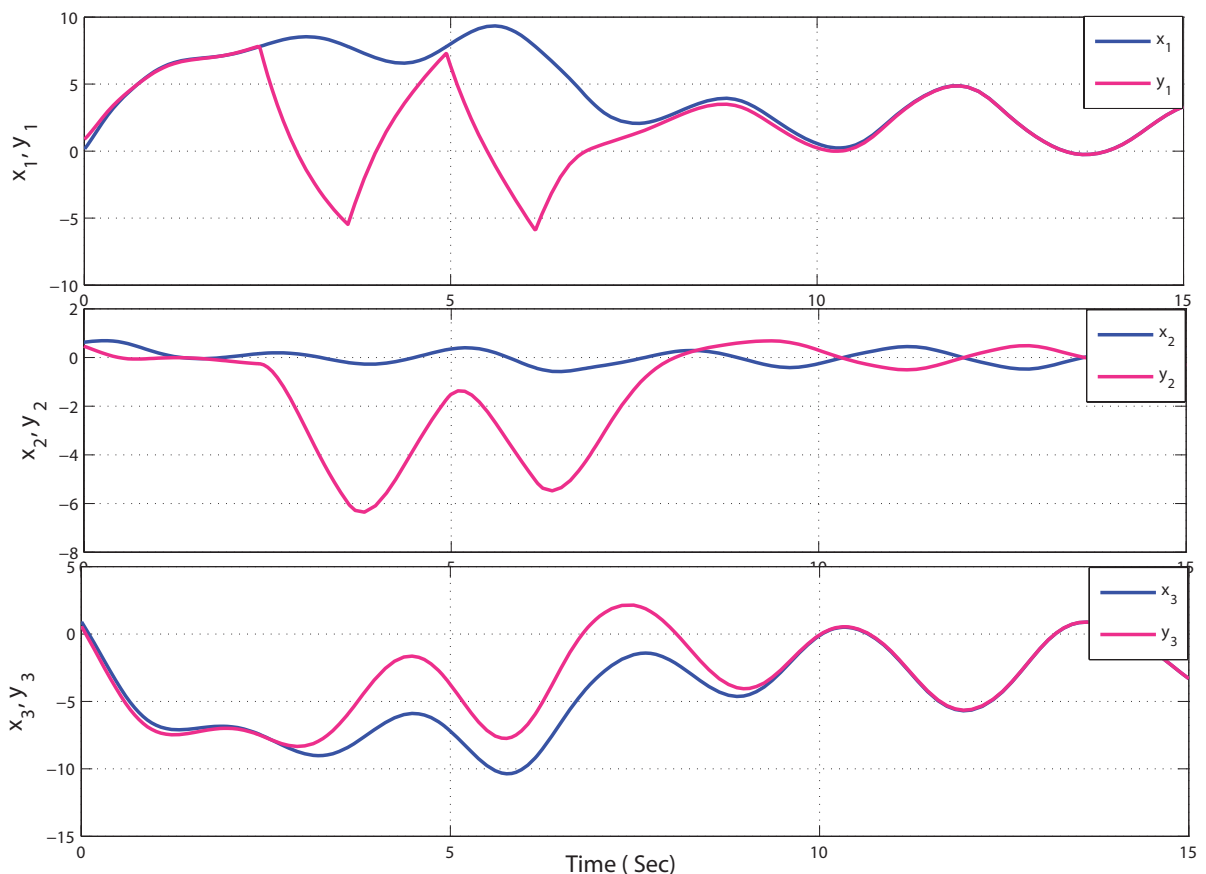

b)

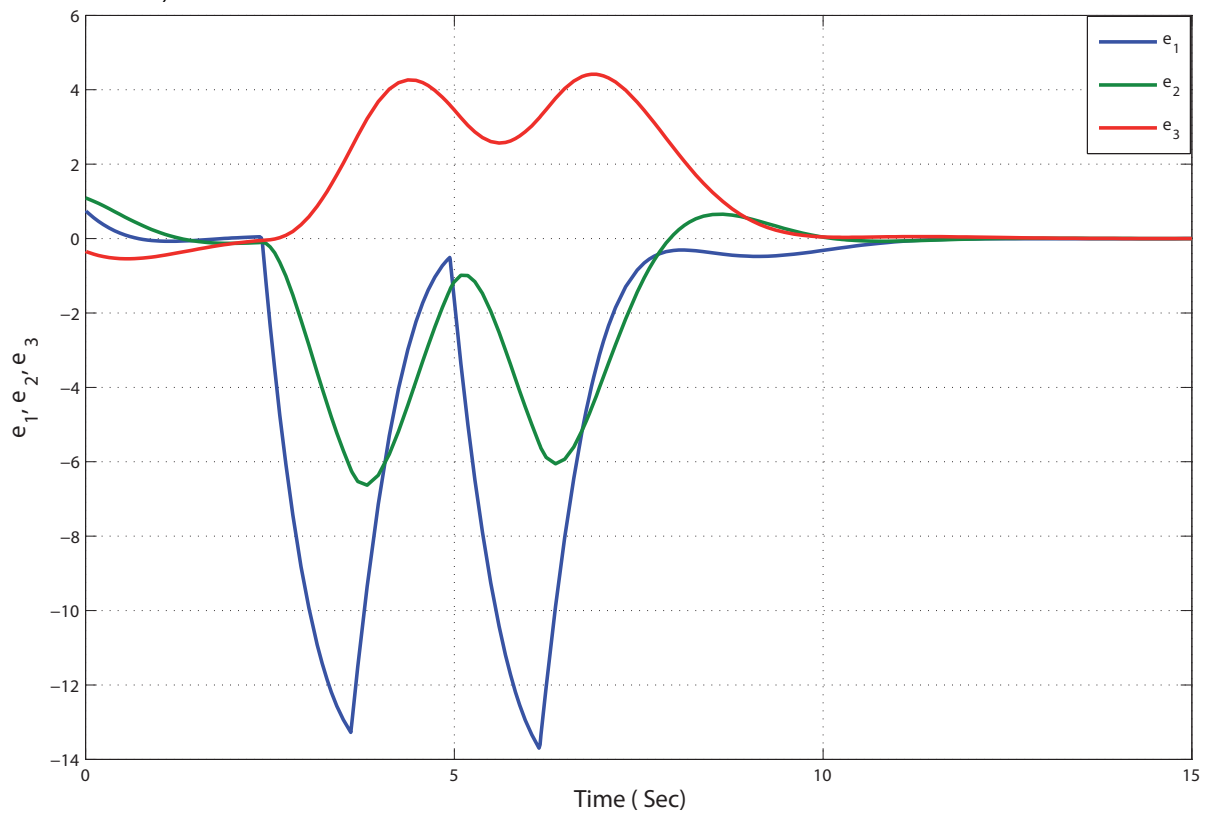

Figure 3. (a). The hybrid synchronization of identical n-scroll Chua's system, (b). Error plot for identical n-scroll Chua's system. 
between the slave system (33) that is to be controlled and the controlling master system (17) as

$$
e_{i}=y_{i} \pm x_{i}, i=1,2,3, \ldots, n \text {. }
$$

The error dynamics is obtained as

$$
\begin{aligned}
& \dot{e}_{1}=y_{2}-x_{2}+u_{1} \\
& \dot{e}_{2}=x_{3}+y_{3}+u_{2} \\
& \dot{e}_{3}=a e_{1}+b\left(y_{2}-x_{2}\right)+c e_{3}+12\left(\phi\left(y_{1}\right)-\phi\left(x_{1}\right)\right)+u_{3}
\end{aligned}
$$

We introduce the backstepping procedure to design the controller $u_{i}, i=1,2,3$, where $u_{i}, i=1,2,3$ are control feedbacks. As long as these feedbacks stabilize system (34) error converges to zero as time goes to infinity.

Firstly we consider the stability of the system

$$
\dot{e}_{1}=y_{2}-x_{2}+u_{1}
$$

where $e_{2}$ is regarded as virtual controller. We consider the Lyapunov function defined by

$$
V_{1}\left(e_{1}\right)=\frac{1}{2} e_{1}^{2}
$$

and its the derivative as follows

$$
\dot{V}_{1}=e_{1}\left(y_{2}-x_{2}+u_{1}\right)
$$

Assume the controller $e_{2}=\alpha_{1}\left(e_{1}\right)$. If

$$
u_{1}=x_{2}-y_{2}-e_{1}+e_{2}
$$

and $\alpha_{1}\left(e_{1}\right)=0$ then

$$
\dot{V}_{1}=-\beta e_{1}^{2}
$$

which is negative definite function. The recursive feedback control $u_{1}$ and $\alpha_{1}\left(e_{1}\right)$ makes the system (35) asymptotically stable. Function $\alpha_{1}\left(e_{1}\right)$ is an estimating function when $e_{2}$ is considered as a controller. The error between $e_{2}$ and $\alpha_{1}\left(e_{1}\right)$ is

$$
w_{2}=e_{2}-\alpha_{1}\left(e_{2}\right)
$$

Consider $\left(e_{1}, w_{2}\right)$ subsystem given by

$$
\begin{aligned}
\dot{e}_{1} & =w_{2}-e_{1} \\
\dot{w}_{2} & =x_{3}+y_{3}+u_{2}
\end{aligned}
$$

and $e_{3}$ as a virtual controller in system (39). Assume that it is equal to $\alpha_{2}\left(e_{3}, w_{2}\right)$ and it makes system (39) asymptotically stable. Consider the Lyapunov function defined by

$$
V_{2}\left(e_{1}, w_{2}\right)=V_{1}\left(e_{1}\right)+\frac{1}{2} w_{2}^{2}
$$


The derivative of $V_{2}\left(e_{1}, w_{2}\right)$ is

$$
\dot{V}_{2}=-e_{1}^{2}+w_{2}\left(w_{2}+x_{3}+y_{3}+u_{2}\right) .
$$

If

$$
u_{2}=-e_{1}-w_{2}+e_{3}-x_{3}-y_{3}
$$

and $\alpha_{2}\left(e_{1}, w_{2}\right)=0$ then

$$
\dot{V}_{2}=-e_{1}^{2}-w_{2}^{2}
$$

which is negative definite function. The recursive feedback $u_{2}$ and $\alpha_{2}\left(e_{1}, w_{2}\right)$ makes the system (39) asymptotically stable. Define the error variable $w_{3}$ as

$$
w_{3}=e_{3}-\alpha_{2}\left(e_{1}, w_{2}\right) .
$$

Consider $\left(e_{1}, w_{2}, w_{3}\right)$ subsystem given by

$$
\begin{aligned}
\dot{e}_{1} & =w_{2}-e_{1} \\
\dot{w_{2}} & =w_{3}-w_{2}-e_{1} \\
\dot{w_{3}} & =a e_{1}+b\left(y_{2}-x_{2}\right)+c e_{3}+12\left(\phi\left(y_{1}\right)-\phi\left(x_{1}\right)\right)+u_{3} .
\end{aligned}
$$

Consider the Lyapunov function defined by

$$
V_{3}\left(e_{1}, w_{2}, w_{3}\right)=V_{2}\left(e_{1}, w_{2}\right)+\frac{1}{2} w_{3}^{2} .
$$

The derivative of $V_{3}\left(e_{1}, w_{2}, w_{3}\right)$ is

$$
\begin{aligned}
\dot{V}_{3}= & -e_{1}^{2}-w_{2}^{2}+w_{3}\left((a-b-2) e_{1}\right. \\
& +(b-2-2 c+1) w_{2}+(2+c) w_{3} \\
& \left.+12\left(\phi\left(y_{1}\right)-\phi\left(x_{1}\right)\right)+u_{3}\right) .
\end{aligned}
$$

If

$$
\begin{aligned}
u_{3}= & -w_{2}-w_{3}-a e_{1}-b\left(y_{2}-x_{2}\right)-c e_{3} \\
& -12\left(\phi\left(y_{1}\right)-\phi\left(x_{1}\right)\right)
\end{aligned}
$$

then

$$
\dot{V}_{3}=-e_{1}^{2}-w_{2}^{2}-w_{3}^{2}
$$

which is negative definite function. The recursive feedback $u_{3}$ makes the system (43) asymptotically stable.Thus, by Lyapunov stability theory, the error dynamics (34) is globally exponentially stable. Hence, we obtain the following result. 
Theorem 2 The identical Lur'e systems (17) and (33) are globally and exponentially hybrid synchronized with the backstepping controls

$$
\begin{aligned}
u_{1}= & x_{2}-y_{2}-e_{1}+e_{2} \\
u_{2}= & u_{2}=-e_{1}-w_{2}+e_{3}-x_{3}-y_{3} \\
u_{3}= & -w_{2}-w_{3}-a e_{1}-b\left(y_{2}-x_{2}\right)-c e_{3} \\
& -12\left(\phi\left(y_{1}\right)-\phi\left(x_{1}\right)\right)
\end{aligned}
$$

\section{Numerical Simulation}

For the numerical simulations, the fourth order Runge-Kutta method is used to solve the system of differential equations (17) and (33) with the backstepping controls $u_{1}, u_{2}$ and $u_{3}$ given by (47). The parameters of the systems (17) and (33) are taken in the chaotic case as

$$
a=-7.4, b=-4.1, c=-1 . k=3.6 .
$$

The initial value of the master system (17) are chosen as

$$
x_{1}(0)=0.785, x_{2}(0)=0.943, x_{3}(0)=0.212
$$

and slave system (33) are chosen as

$$
y_{1}(0)=0.253, y_{2}(0)=0.558, y_{3}(0)=0.756
$$

Fig. 4 (a) and (b) depict the synchronization of identical Lur'e systems (17) and (33).

\section{The hybrid synchronization of n-scroll Chua system and Lur'e system via backstepping control design with novel feedback}

In this section we apply the backstepping method with novel feedback function for the hybrid synchronization of n-scroll Chua [40] and Lur'e systems [41, 42]. The master system is described by the chaotic n-scroll Chua's system dynamics (15) with parameters (16).

The slave system is described by Lur'e system as follows

$$
\begin{aligned}
& \dot{y}_{1}=y_{2}+u_{1} \\
& \dot{y}_{2}=y_{3}+u_{2} \\
& \dot{y}_{3}=\eta y_{1}+\theta y_{2}+\lambda y_{3}+12 \phi\left(y_{1}\right)+u_{3}
\end{aligned}
$$

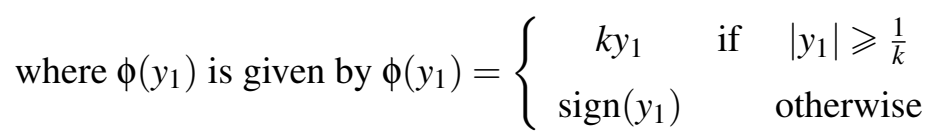



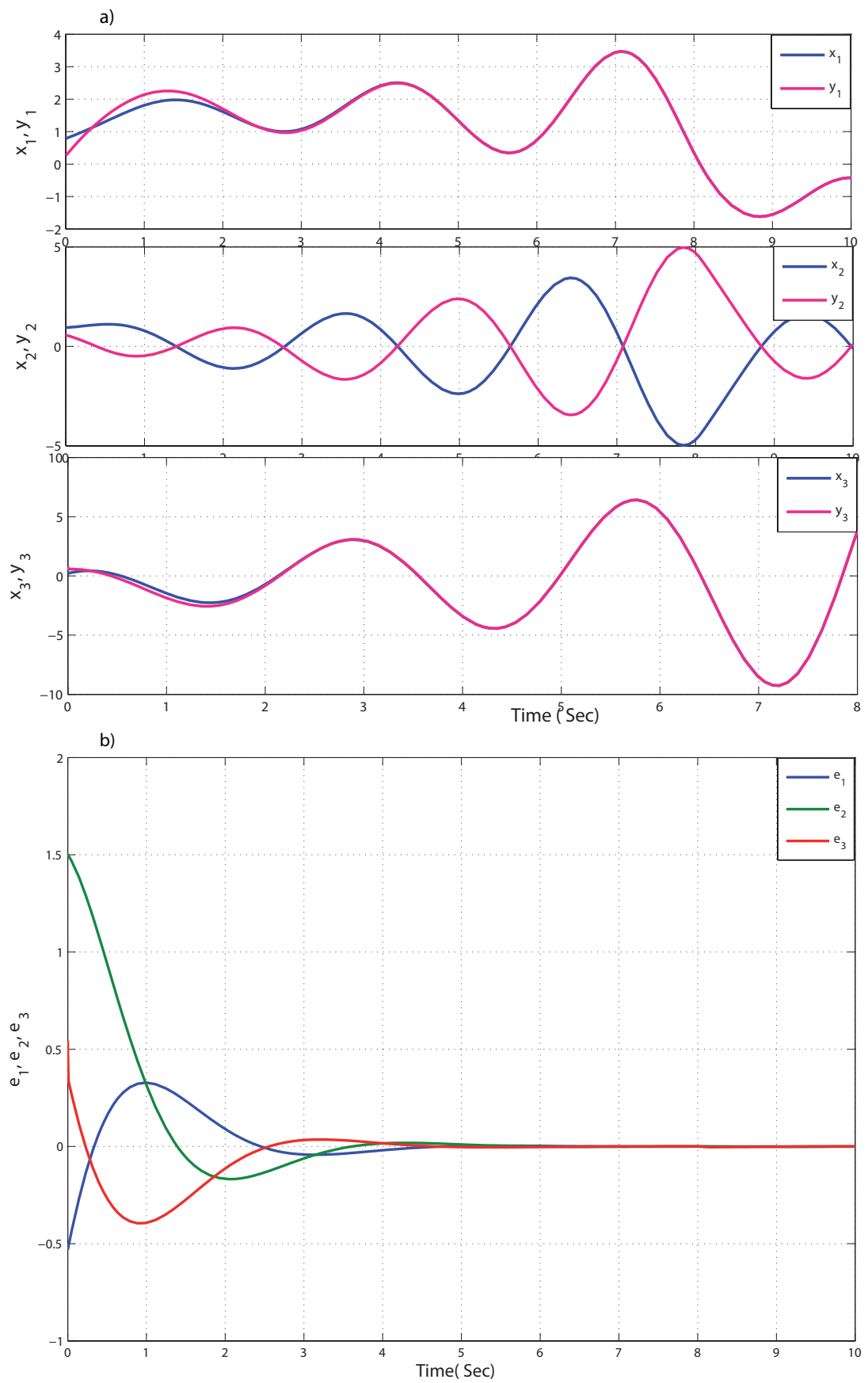

Figure 4. (a). The hybrid synchronization of identical Lur'e system, (b). Error plot for identical Lur'e system. 
where $y(t)(i=1,2,3) \in \mathbb{R}^{3}$ is a state vectors of the system, $\eta, \theta, \lambda$ and $k$ are positive real constants. When

$$
\eta=-7.4, \theta=-4.1, \lambda=-1, k=3.6
$$

the chaotic attractors are generated. Let us define the error variables between the slave system (48) that is to be controlled and the controlling master system (15) as

$$
e_{i}=y_{i} \pm x_{i}, i=1,2,3, \ldots, n \text {. }
$$

The error dynamics is obtained as

$$
\begin{aligned}
& \dot{e}_{1}=y_{2}-\alpha\left(x_{2}-f\left(x_{1}\right)\right)+u_{1} \\
& \dot{e}_{2}=y_{3}+x_{1}-x_{2}+x_{3}+u_{2} \\
& \dot{e}_{3}=\eta y_{1}+\theta y_{2}+\lambda y_{3}+12 \phi\left(y_{1}\right)+\beta x_{2}+u_{3}
\end{aligned}
$$

We introduce the backstepping procedure to design the controller $u_{i}, i=1,2,3$ where $u_{i}, i=1,2,3$ are control feedbacks. As long as these feedbacks stabilize system (49), the error converge to zero as the time $t$ goes to infinity. Firstly we consider the stability of the system

$$
\dot{e}_{1}=y_{2}-\alpha\left(x_{2}-f\left(x_{1}\right)\right)+u_{1}
$$

where $e_{2}$ is regarded as virtual controller. We consider the Lyapunov function defined by

$$
V_{1}\left(e_{1}\right)=\frac{1}{2} e_{1}^{2}
$$

The derivative of $V_{1}$ is as follows

$$
\dot{V}_{1}=e_{1}\left(y_{2}-\alpha\left(x_{2}-f\left(x_{1}\right)\right)+u_{1}\right) \text {. }
$$

Assume the controller $e_{2}=\alpha_{1}\left(e_{1}\right)$. If $\alpha_{1}\left(e_{1}\right)=0$ and the feedback input

$$
u_{1}=-e_{1}+e_{2}-y_{2}+\alpha\left(x_{2}-f\left(x_{1}\right)\right)
$$

then

$$
\dot{V}_{1}=-e_{1}^{2}
$$

which is negative definite function. The recursive feedback $u_{1}$ and $\alpha_{1}\left(e_{1}\right)$ makes the system (50) asymptotically stable. Function $\alpha_{1}\left(e_{1}\right)$ is an estimating function when $e_{2}$ is considered as a controller. The error between $e_{2}$ and $\alpha_{1}\left(e_{1}\right)$ is

$$
w_{2}=e_{2}-\alpha_{1}\left(e_{1}\right)
$$

Consider $\left(e_{1}, w_{2}\right)$ subsystem given by

$$
\begin{aligned}
\dot{e}_{1} & =-e_{1}+e_{2} \\
\dot{w}_{2} & =y_{3}+x_{1}-x_{2}+x_{3}+u_{2}
\end{aligned}
$$


and $e_{3}$ as a virtual controller in system (54). Assume that the controller equal to $\alpha_{2}\left(e_{3}, w_{2}\right)$ makes system (54) asymptotically stable. Consider the Lyapunov function defined by

$$
V_{2}\left(e_{1}, w_{2}\right)=V_{1}\left(e_{1}\right)+\frac{1}{2} w_{2}^{2} .
$$

The derivative of $V_{2}\left(e_{1}, w_{2}\right)$ is

$$
\dot{V}_{2}=-e_{1}^{2}+w_{2}\left(e_{1}+y_{3}+x_{1}-x_{2}+x_{3}+u_{2}\right) .
$$

If $e_{3}=\alpha_{2}\left(e_{1}, w_{2}\right)=0$ and the feedback input

$$
u_{2}=-w_{2}-e_{1}-y_{3}-x_{1}+x_{2}-x_{3}+e_{3}
$$

then

$$
\dot{V}_{2}=-e_{1}^{2}-w_{2}^{2}
$$

which is negative definite function. The recursive feedback $u_{2}$ and $\alpha_{2}\left(e_{1}, w_{2}\right)$ make the system (54) asymptotically stable. Define the error variable $w_{3}$ as

$$
w_{3}=e_{3}-\alpha_{2}\left(e_{1}, w_{2}\right) .
$$

Consider $\left(e_{1}, w_{2}, w_{3}\right)$ subsystem given by

$$
\begin{aligned}
\dot{e_{3}} & =-e_{1}+w_{2} \\
\dot{w_{2}} & =w_{3}-e_{1}-w_{2} \\
\dot{w_{3}} & =\eta y_{1}+\theta y_{2}+\lambda y_{3}+12 \phi\left(y_{1}\right)+\beta x_{2}+u_{3} .
\end{aligned}
$$

Consider also the Lyapunov function defined by

$$
V_{3}\left(e_{3}, w_{2}, w_{3}\right)=V_{2}\left(e_{1}, w_{2}\right)+\frac{1}{2} w_{3}^{T} w_{3} .
$$

The derivative of $V_{3}\left(e_{3}, w_{2}, w_{3}\right)$ is

$$
\begin{aligned}
\dot{V}_{3}= & -e_{1}^{2}-\left(w_{2}^{2}\right)+w_{3}\left(w_{2}+\eta y_{1}+\theta y_{2}+\lambda y_{3}\right. \\
& \left.+12 \phi\left(y_{1}\right)+\beta x_{2}+u_{3}\right) .
\end{aligned}
$$

If

$$
u_{3}=-w_{2}-\eta y_{1}-\theta y_{2}-\lambda y_{3}-12 \phi\left(y_{1}\right)-\beta x_{2}-w_{3}
$$

then

$$
\dot{V}_{3}=-e_{1}^{2}-\left(w_{2}^{2}\right)-w_{3}^{2}
$$

which is negative definite function. The recursive feedback control $u_{3}$ makes the system (58) asymptotically stable. Thus, by Lyapunov stability theory, the error dynamics (49) is globally exponentially stable. Hence, we obtain the following result. 
Theorem 3 The n-scroll Chua's system (15) and Lur'e chaotic system (48) are globally and exponentially hybrid synchronized with the backstepping controls design with feedback inputs

$$
\begin{aligned}
& u_{1}=-e_{1}+e_{2}-y_{2}+\alpha\left(x_{2}-f\left(x_{1}\right)\right) \\
& u_{2}=-w_{2}-e_{1}-y_{3}-x_{1}+x_{2}-x_{3}+e_{3} \\
& u_{3}=-w_{2}-\eta y_{1}-\theta y_{2}-\lambda y_{3}-12 \phi\left(y_{1}\right)-\beta x_{2}-w_{3} .
\end{aligned}
$$

\section{Numerical simulation}

For the numerical simulations, the fourth order Runge-Kutta method is used to solve system of differential equations (15) and (48) with the backstepping controls $u_{1}, u_{2}$ and $u_{3}$ given by (61). The parameters of the systems (15) and (48) are taken in the chaotic case as

$$
\alpha=10.814, \beta=14.0, a=1.3, b=0.11, c=3, d=0
$$

and

$$
\eta=-7.4, \theta=-4.1, \lambda=-1 ; k=3.6 .
$$

The initial value of the master system (15) are chosen as

$$
x_{1}(0)=0.012, x_{2}(0)=0.764, x_{3}(0)=0.064
$$

and slave system (48) are chosen as

$$
y_{1}(0)=0.984, y_{2}(0)=0.231, y_{3}(0)=0.999
$$

Fig. 5 (a) and (b) depict the hybrid synchronization of n-scroll Chua and Lur'e chaotic systems (15) and (49).

\section{Conclusion}

In this paper, backstepping control method based on Lyapunov stability theory has been applied to achieve global chaos hybrid synchronization for the n-scroll Chua and Lur'e chaotic systems. The advantage of this method is that it follows a systematic procedure for hybrid synchronizing chaotic system and there is no derivative in the controller. The backstepping control design has been demonstrated on $n-$ scroll Chua and Lur'e chaotic systems. Numerical simulations have been given to illustrate and validate the effectiveness of the proposed hybrid synchronization schemes for identical n-scroll Chua and Lur'e chaotic systems and non-identical n-scroll Chua and Lur'e chaotic systems. The backstepping method is very effective and convenient to achieve global chaos hybrid synchronization. 


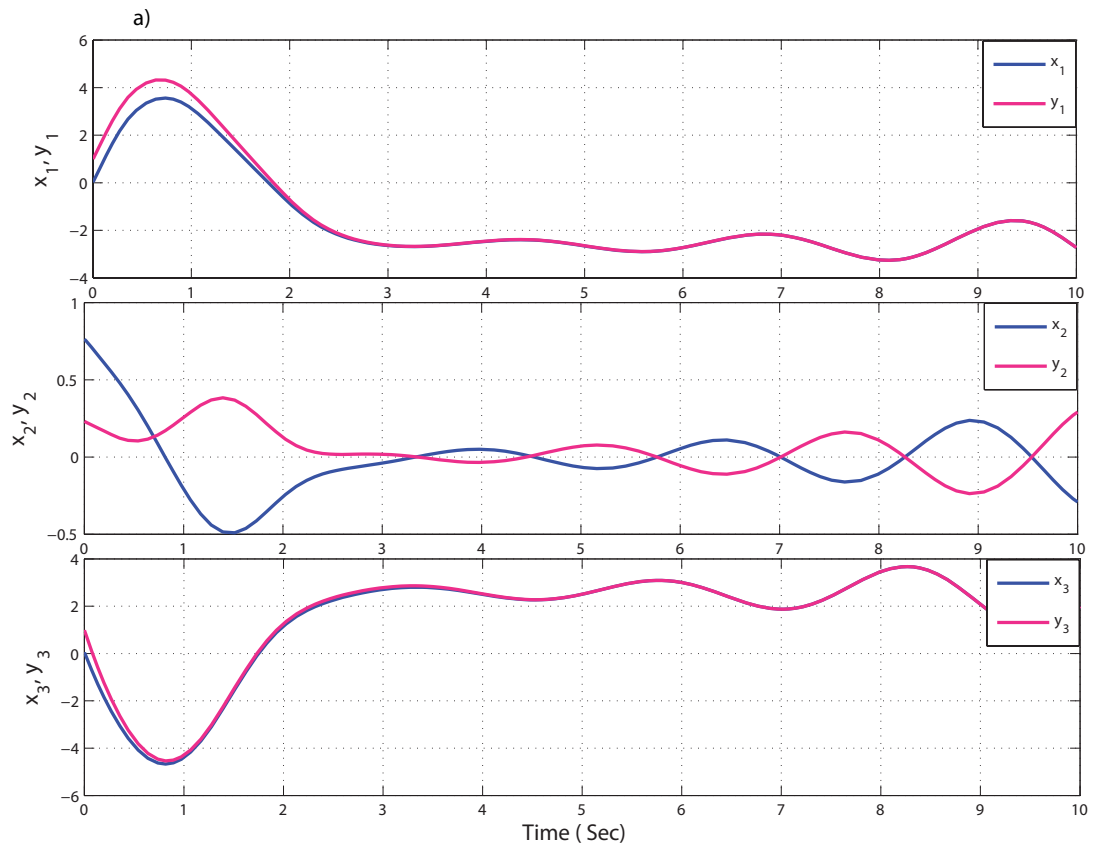

b)

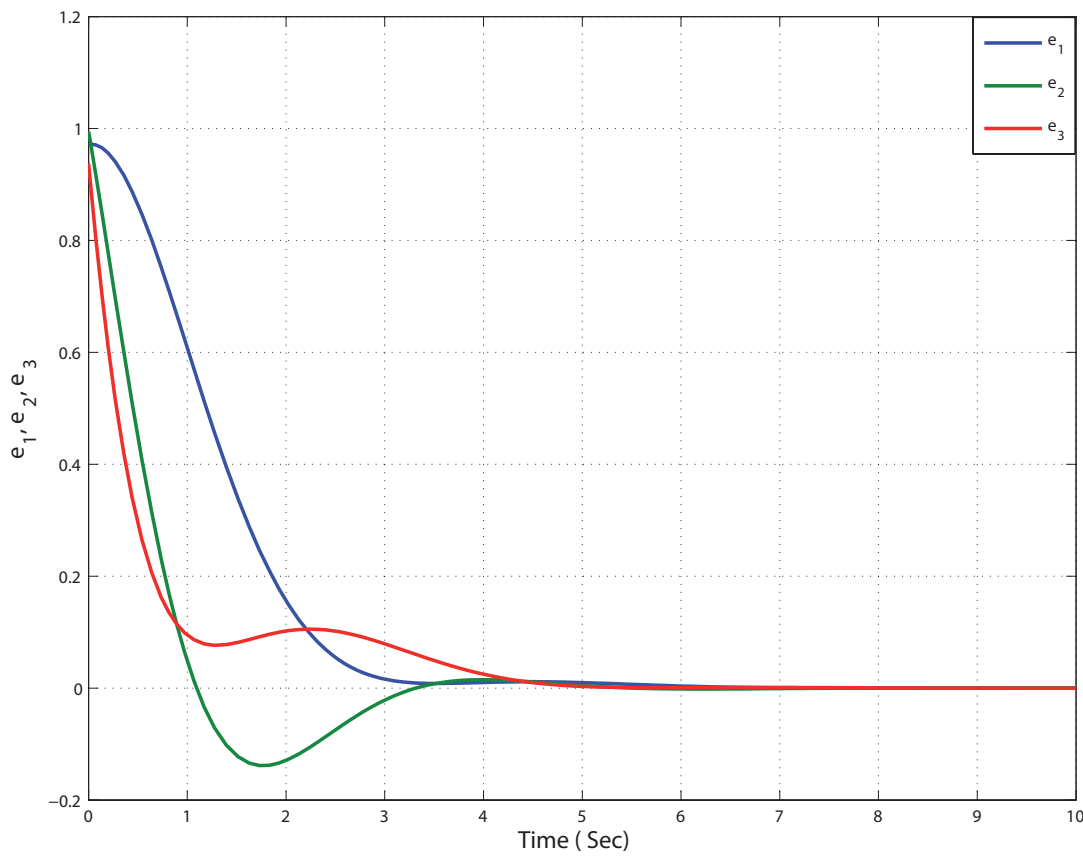

Figure 5. (a). The Hybrid Synchronization of n-scroll Chua's and Lur'e chaotic systems, (b). Error plot for n-scroll Chua's and Lur'e chaotic systems. 


\section{References}

[1] H. FuJisaka and T. Yamada: Stability theory of synchronized motion in coupled-oscillator systems. Progress of Theoretical Physics, 63 (1983), 32-47.

[2] L.M. Pecora and T.L. Carroll: Synchronization in chaotic systems. Phys. Rev. Lett., 64 (1990), 821-824.

[3] L.M. PECORA and T.L. CARRolL:L Synchronizing chaotic circuits. IEEE Trans. Circ. Sys., 38 (1991), 453-456.

[4] K.T. Alligood, T. SAuer and J.A. Yorke: Chaos: An Introduction to Dynamical Systems. Berlin, Germany, Springer Verlag, 1997.

[5] E. Oтт: Chaos in Dynamical Systems. United Kingdom, Cambridge University Press, 2002.

[6] Y.M. WANG and H. ZHU: Generalized synchronization of continuous chaotic systems. Chaos, Solitons and Fractals, 27 (2006), 97-101.

[7] Z.M. GE and C.C. ChEN: Phase synchronization of coupled chaotic multiple time scales systems. Chaos, Solitons and Fractals, 20 (2004), 639-647.

[8] J. QIANG: Projective synchronization of a new hyper chaotic Lorenz systems. Phys. Lett. A, 370 (2007), 40-45.

[9] Y. JIAN-PING and L. CHANG-PIN: Generalized projective synchronization for the chaotic Lorenz systems and the chaotic Chen system. J. of Shanghai University, 10 (2006), 299-304.

[10] R. H. LI, W. XU and S. LI: Adaptive generalized projective synchronization in different chaotic systems based on parameter identifiction. Phys. Lett. A, 367 (2007), 199-206.

[11] V. SUNDARAPANDIAN and P. SARASU: Generalized projective synchronization of double-scroll chaotic systems using active feedback control. CCSIT 2012, Part-I, LNICST 84, Springer Heldelberg, (2012), 111-118.

[12] P. SARAsu and V. Sundarapandian: Generalized projective synchronization of three-scroll chaotic systems via. active control. CCSIT 2012, Part-I, LNICST 84, Springer Heldelberg, (2012), 124-133.

[13] R.H. LI: A special full-state hybrid projective synchronization in symmetrical chaotic systems. Applied Math. Comput., 200 (2008), 321-329.

[14] V. Sundarapandian and R. SuRESH: Hybrid synchronization of Arneodo and Rössler chaotic systems by active nonlinear control. CCSIT 2012, Part-I, LNICST, 84 Springer Heldelberg, (2012), 257-266. 
[15] V. Sundarapandian and S. SivaPerumal: Hybrid synchronization of hyper chaotic Chen system via sliding mode control. CCSIT 2012, Part-I, LNICST, 84 Springer Heldelberg, (2012), 73-82.

[16] K. Murali and M. Lakshmanan: Secure communication using a compound signal using sampled-data feedback. Applied Mathematics and Mechanics, 11 (2003), 1309-1315.

[17] T. YANG and L.O. ChUA: Generalized synchronization of chaos via linear transformations. Internat. J. Bifur. Chaos, 9 (1999), 215-219.

[18] K. Murali and M. LaKshmanan: Chaos in Nonlinear Oscillators: Controlling and Synchronization. Singapore: World Scientific, 1996.

[19] S.K. Han, C. Kerrer and Y. Kuramoto: D-phasing and bursting in coupled neural oscillators. Phys. Rev. Lett., 75 (1995), 3190-3193.

[20] B. Blasius, A. Huppert and L. Stone: Complex dynamics and phase synchronization in spatially extended ecological system. Nature, 399 (1999), 354-359.

[21] L. KocAREV and U. PARLITZ: General approach for chaotic synchronization with applications to communications. Phys. Rev. Lett., 74 (1995), 5028-5030.

[22] Z. WANG: Chaos synchronization of an energy resource system based on linear control. Nonlinear Analysis: Real world Application. (in press).

[23] J. WAng, L. Chen, B. Deng: Synchronization of ghostburster neurons in external electrical stimulation via $H_{\infty}$ variable universe fuzzy adaptive control. Chaos, Solitons and Fractals, 39 (2009), 2076-2085.

[24] F.M. Moukam Kakmeni, J.P. Nguenang and T.C. Kofane: Chaos synchronization in bi-axial magnets modeled by bloch equation modeled by Bloch equations. Chaos, Solitons and Fractals, 30 (2006), 690-699.

[25] J.L. HINDMARSH and R.M. RosE: A model of neuronal bursting using 3-coupled 1 st order differential equations. Proc. Roy. Soc. Lond. B. Biol., 221 (1984), 81-102.

[26] Y.-Q. Che, J. WANG, K.-M. TsANG and W.-L. Chen: Unidirectional synchronization for Hindmarsh-Rose neurons via robust adaptive sliding mode control. Nonlinear Analysis: Real world Application, 11 (2010), 1096-1104.

[27] G. Zhao Zeng, L.S. Chen and L.H. Sun: Complexity of an SIR epidemic dynamics model with impulsive vaccination control. Chaos, Solitons and Fractals, 26 (2005), 495-505.

[28] J. WANG, D. LU and L. TIAN: Global synchronization for time delay WINDMI system. Chaos, Solitons and Fractals, 30 (2006), 629-635. 
[29] E. Ott, C. Grebogi and J.A. Yorke: Controlling chaos. Phys. Rev. Lett., 64 (1990), 1196-1199.

[30] J.H. PARK and O.M. KWON: A novel criterion for delayed feedback control of time-delay chaotic systems. Chaos, Solitons and Fractals, 17 (2003), 709-716.

[31] J. LU, X. WU, X. HAN and J. LU: Adaptive feedback synchronization of a unified chaotic system. Phys. Lett. A, 329 (2004), 327-333.

[32] J.H. PARK, S.M. LEE and O.M. KwON: Adaptive synchronization of GenesioTesi chaotic system via a novel feedback control. Phys. Lett. A, 371 (2007), 263270.

[33] J.H. PARK: Adaptive control for modified projective synchronization of a fourdimensional chaotic system with uncertain parameters. J. Computational and Applied Math., 213 (2008), 288-293.

[34] H.T. YAU: Design of adaptive sliding mode controller for chaos synchronization with uncertainties. Chaos, Solitons and Fractals, 22 (2004), 341-347.

[35] V. Sundarapandian: Global chaos synchronization of the Pehlivan systems by sliding mode control. Int. J. Comp. Sci. and Engn., 3 (2011), 2163-2169.

[36] V. SUNDARAPANDIAN and R. SURESH: Global chaos synchronization for Rossler and Arneodo chaotic systems by nonlinear control. Far East Journal of Applied Mathematics, 44 (2010), 137-148.

[37] V. Sundarapandian and R. SuResh: New results on the global chaos synchronization for Liu-Chen-Liu and Lü chaotic systems. PEIE 2010, CCIS, 102 Springer-Verlag Berlin Heidelberg, (2010), 20-27.

[38] X. WU and J. LU: Parameter identification and backstepping control of uncertain Lu system. Chaos, Solitons and Fractals, 18 (2003), 721-729.

[39] Y.G. YU and S.C. ZHANG Adaptive backstepping synchronization of uncertain chaotic systems. Chaos, Solitons and Fractals, 27 (2006), 1369-1375.

[40] K.S. Wallace, G. Tang, Q.Zhong, G. Chen and K.F. Man: Generation of $\mathrm{n}$-scroll attractors via sine function. IEEE Trans. Circ. Sys-I Fundamental Theory and Applications, 48 (2001), 1369-1372.

[41] J. Suykens, J. VAndewalle and L. Chua: Nonlinear $H_{\infty}$ synchronization of chaotic Lur'e system. Int. J. Bifurcation Chaos, 7 (1997), 1323-1325.

[42] J. Suykens, J. Vandewalle and L. Chua: Robust synthesis for master-slave synchronization of Lur'e system. IEEE Trans. Circ. Sys-I, 46 (1999), 841-850.

[43] W. HAHN: The Stability of Motion. Berlin, Germany, Springer-Verlag, 1967. 